\title{
An extension of Lucas identity via Pascal's triangle
}

\author{
Giuseppina Anatriello*1 ${ }^{*}$, Giovanni Vincenzi² ${ }^{\circledR}$ \\ ${ }^{1}$ Dipartimento di Architettura, Università di Napoli "Federico II", Napoli, Italy \\ ${ }^{2}$ Dipartimento di Matematica, Università di Salerno, Via Giovanni Paolo II, Fisciano, Salerno, Italy
}

\begin{abstract}
The Fibonacci sequence can be obtained by drawing diagonals in a Pascal's triangle, and from this, we can obtain the Lucas identity. An investigation on the behavior of certain kinds of other diagonals inside a Pascal's triangle identifies a new family of recursive sequences: the $k$-Padovan sequences. This family both contains the Fibonacci and the Padovan sequences. A general binomial identity for $k$-Padovan sequences which extends both the well-known Lucas identity and the less known Padovan identity is derived.
\end{abstract}

Mathematics Subject Classification (2020). Primary 05A19; Secondary 65Q30, 11Y55, 03D20

Keywords. combinatorial identities, Pascal's triangle, $k$-Fibonacci diagonals, Fibonacci sequence, Padovan sequence

\section{Introduction}

In number theory, the study of recursive sequences and related identities by binomial coefficients has a certain relevance (see $[1,9,10,19,22,24]$, and references therein). One of the most famous result in this context is probably the "Lucas identity" by the name of the great French mathematician François Édouard Anatole Lucas (1841-1891) who discovered it. He proved that each term $F_{m}$ of the Fibonacci sequence $\left(F_{m}\right)$ can be expressed as a sum of binomials (see for instance [5], p.112) as follows:

$$
F_{m}=\left(\begin{array}{c}
m-1 \\
0
\end{array}\right)+\underbrace{\left(\begin{array}{c}
m-2 \\
1
\end{array}\right)+\ldots+\left(\begin{array}{c}
m-1-i \\
i
\end{array}\right)+\ldots}_{\lfloor m-1 / 2\rfloor},
$$

where $\lfloor m-1 / 2\rfloor$ represents the integer part of $m$.

Similar relations also hold for other relevant sequences.

The Cordonnier sequence 1, 1, 1,2,2,3,4,5,7,9,12 .., better known as Padovan sequence (see [21]), is a linear homogeneous recurrence with constant coefficients defined as:

$$
\left\{\begin{array}{l}
P_{1}=P_{2}=P_{3}=1 \\
P_{m}=P_{m-2}+P_{m-3}, \quad m>3 .
\end{array}\right.
$$

\footnotetext{
*Corresponding Author.

Email addresses: anatriello@unina.it (G. Anatriello), vincenzi@unisa.it (G. Vincenzi)

Received: 28.05.2020; Accepted: 09.10.2020
} 

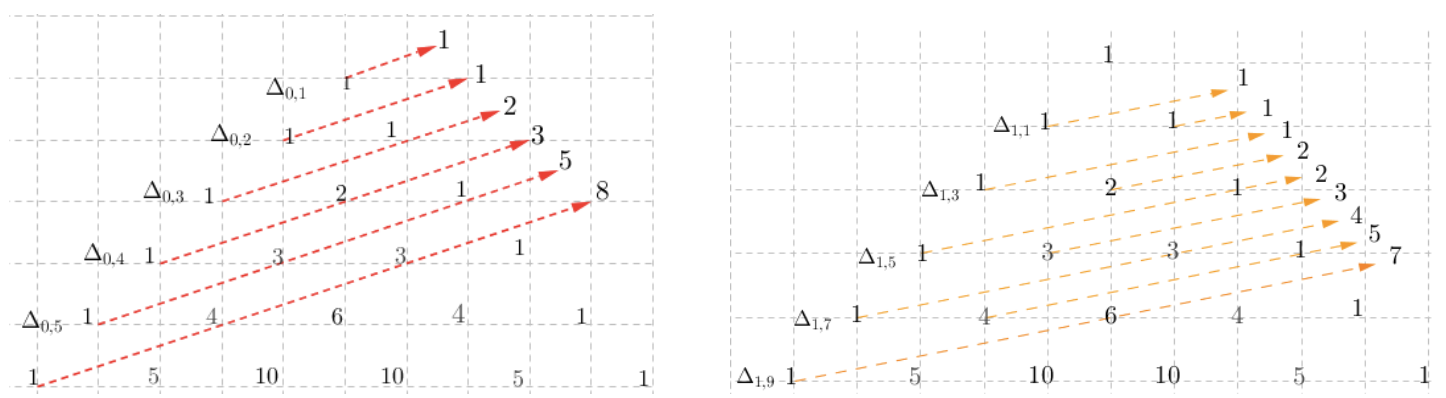

Figure 1. In a Pascal's triangle, the Fibonacci and the Padovan numbers can be obtained as the associated terms to 'Fibonacci' and the 'Padovan' diagonals, respectively. Note that the first Fibonacci diagonal, $\Delta_{0,1}$, starts from the vertex (the 0-row); while the first Padovan diagonal $\Delta_{1,1}$, starts from the 1-row of a Pascal's triangle.

This sequence has many interesting properties (see $[3,7,16,23]$ and references therein), in particular its 'Kepler limit', that is the limit of the ratio of consecutive terms (see $[6,8]$ ), is the so-called Plastic number. This number has a certain relevance in contemporary Architecture (see $[12,16,17]$ ), and it is considered the key to finding the right proportion among spatial figures at the same way that the Golden Mean is considered for the flat ones (see [15]).

It is known, even if not well-highlighted in literature (see $[3,16,17,22]$ ), that each term $P_{m}$ of the Padovan sequence can be described as sum of binomial coefficients. In fact, the following relation also known as Padovan identity holds:

$$
P_{m}=\sum_{i=0}^{\lfloor n / 3\rfloor}\left(\begin{array}{c}
n-i \\
2 i
\end{array}\right) \text { if } m=2 n-1 \text { and } P_{m}=\sum_{i=0}^{\lfloor n / 3\rfloor}\left(\begin{array}{c}
n-i \\
2 i+1
\end{array}\right) \text { if } m=2 n .
$$

Therefore, both Fibonacci and Padovan numbers can be written as sum of binomial coefficients of particular kind, we could say 'consecutive'. It is well-known that both the Lucas and the Padovan identities (1.1) and (1.3) have a visual interpretation by a Pascal's triangle (see Figure 1).

It can be noted that all Fibonacci diagonals, $\Delta_{0,1}, \ldots, \Delta_{0, m}, \ldots$, have the same inclination (Fib-inclination), which is different from the Pad-inclination of Padovan diagonals $\Delta_{1,1}, \ldots, \Delta_{1, m} \ldots$, as Figure 1 shows.

To find numerical identities inside geometric configurations is a classical mathematical topic (see for example, $[2,13,14,20]$ and references therein), so that it is natural to consider other diagonals with an increasing $(k)$-inclination (see Figure 2 ): the $k$-Fibonacci diagonals denoted by

$$
\left(\Delta_{k, m}\right):=\Delta_{k, 1}, \ldots, \Delta_{k, m} \ldots,
$$

and their associated terms $S_{k, m}$ defined as the sum of the elements which lie on them. (We note that the $k$-diagonals are parallel to ray $(k+1,1)$ of [4]). Two natural questions arise:

1 Is $\left(S_{k, m}\right)$ a recursive sequence?

2 If $\left(S_{k, m}\right)$ is a recursive sequence for every $k \geq 0$, is there a general binomial identity which represents $S_{k, m}$ ?

In this paper, we will give a positive answer to both the questions.

The paper is organized as follows. In Section 2, we investigate the properties of the $k$-Fibonacci diagonals of a Pascal's triangle and their associated terms $S_{k, m}$, which are expressed as the sum of binomials. In Section 3, we introduce a family of recursive linear 


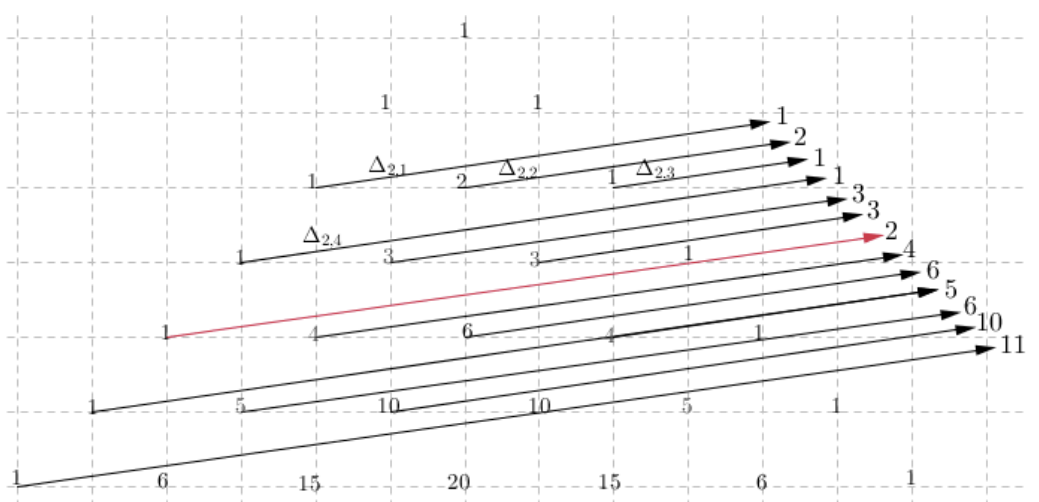

Figure 2. The sequence of diagonals $\left(\Delta_{2, m}\right)$ in a Pascal's triangle $\mathcal{T}$, and the sequence of their associated terms $S_{2, m}: 1,2,1,1,3,3,2,4,6,5,6,10,11, \ldots$.

homogenous sequences with constant coefficients, the $k$-Padovan sequences $\left(P_{k, m}\right)$. The main theorem shows that $S_{k, m}=P_{k, m}$ and at the same time a general binomial identity for $k$-Padovan $\left(P_{k, m}\right)$ sequences is established in Theorem 3.4. As special cases Lucas identity and Padovan identity can been derived (see Remarks 3.5 and 3.6).

\section{The $k$-Fibonacci diagonals of a Pascal's triangle}

In this section, as usual, by the term "diagonal" we mean at the same time something of geometric (a line) and discrete (the elements which lie above it) object.

Given a whichever configuration of numbers disposed in triangular way, say $T$, for any $i \geq 0$ and $0 \leq j \leq i$ we will denote by $Q(i, j)$ the $(i, j)$ entry of $T$, that is the element that lies on the $j$ site (counting from the left to the right, starting from 0 ) of the $i$-th row of $T$. The top of the triangle is considered as the 0 -row. For example if $\mathcal{T}$ is the classical Pascal's triangle (see Figure 1 ) we have $Q(0,0)=1, Q(3,0)=1, Q(3,1)=3$, and more generally we have $Q(i, j)=\left(\begin{array}{l}i \\ j\end{array}\right)$.

It can be noted that the inclination of Fibonacci diagonals (Figure 1 on the left) is slightly smaller than the inclination of Padovan diagonals (Figure 1 on the right). Precisely, if an element, say $Q(i, j)$, lies on a Fibonacci diagonal, then the element above on the same diagonal shifts of just one position, so it is $Q(i-1, j+1)$; while if an element, say $Q(i, j)$, lies on a Padovan diagonal, then the element above on the same diagonal shifts of two positions, so it is $Q(i-1, j+2)$.

So that, for every positive integer $m$, the classical Fibonacci diagonals $\Delta_{0, m}$ and the sum of the elements lying on each of them, their associated terms $S_{0, m}$, are:

$\Delta_{0,1}=\{Q(0,0)\}$, which gives

$\Delta_{0,2}=\{Q(1,0)\}$, which gives

$\Delta_{0,3}=\{Q(2,0), Q(1,1)\}$, which gives

$\Delta_{0,4}=\{Q(3,0), Q(2,1)\}$, which gives

$\Delta_{0,5}=\{Q(4,0), Q(3,1), Q(2,2)\}$ which gives

$\Delta_{0,6}=\{Q(5,0), Q(4,1), Q(3,2)\}$ which gives

$$
\begin{aligned}
S_{0,1} & =1=F_{1} \\
S_{0,2} & =1=F_{2} \\
S_{0,3}=1+1=2 & =F_{3} \\
S_{0,4}=1+2 & =3=F_{4} \\
S_{0,5}=1+3+1 & =5=F_{5} \\
S_{0,6}=1+4+3 & =8=F_{6}
\end{aligned}
$$


Similarly, the sequence of Padovan diagonals $\Delta_{1, m}$ and their associated terms $S_{1, m}$, are:

$$
\begin{aligned}
& \Delta_{1,1}=\{Q(1,0)\}, \text { which gives } \\
& \Delta_{1,2}=\{Q(1,1)\}, \text { which gives } \\
& \Delta_{1,3}=\{Q(2,0)\}, \text { which gives } \\
& \Delta_{1,4}=\{Q(2,1)\}, \text { which gives } \\
& \Delta_{1,5}=\{Q(3,0), Q(2,2)\}, \text { which gives } \\
& \Delta_{1,6}=\{Q(3,1)\} \text { which gives }
\end{aligned}
$$

$$
\begin{aligned}
S_{1,1} & =1=P_{1} \\
S_{1,2} & =1=P_{2} \\
S_{1,3} & =1=P_{3} \\
S_{1,4} & =2=P_{4} \\
S_{1,5}=1+1 & =2=P_{5} \\
S_{1,6} & =3=P_{6}
\end{aligned}
$$

More generally, in a Pascal's triangle $\mathcal{T}$, just increasing the inclination we may draw the $k$-Fibonacci diagonals for every integer $k \geq 0$. In fact, chosen $k$, we may consider as 'first' $k$-Fibonacci diagonal $\Delta_{k, 1}:=\{Q(k, 0\}$. This means that the triangle on the top of $\mathcal{T}$, consisting of the rows $0,1, \ldots, k-1$ is not crossed by any $k$-diagonal (see for example Figure 2); moreover if an element, say $Q(i, j)$, lies on a $k$-Fibonacci diagonal, then the element above on the same diagonal (if any) shifts on the right of $k+1$ positions, so it is $Q(i-1, j+k+1)$ (see Figure 2).

Clearly, the 0-Fibonacci diagonals are the classical Fibonacci diagonals, and the 1Fibonacci diagonals are exactly the Padovan diagonals.

In Figure 2 some 2-Fibonacci diagonals $\Delta_{2,1}, \Delta_{2,2} \ldots$ are drawn. Their associated terms $S_{2,1}, S_{2,2}, \ldots$ are:

$$
1,2,1,1,3,3,2,4,6,5,6,10,11, \ldots
$$

We can note that the sequence $\left(S_{2, m}\right)$ showed by (2.1) appears to be recursive, linear and homogenous of order 4; its characteristic polynomial being $f=x^{4}-x-1$ (note that two powers of $x$ - namely $x^{2}$ and $x^{3}$ - have zero coefficients), and 'initial conditions' being $\left(\begin{array}{l}2 \\ 0\end{array}\right),\left(\begin{array}{l}2 \\ 1\end{array}\right),\left(\begin{array}{l}2 \\ 2\end{array}\right), 1$.

We can observe that all $k$-Fibonacci diagonals of the same type (same $k$ ) are parallel lines (see Figure 1 and Figure 2); instead of $k \neq k^{\prime}$, where any $k$-Fibonacci diagonals will be not parallel to each $k^{\prime}$-Fibonacci diagonals, as they have different inclinations (see Figure 3).

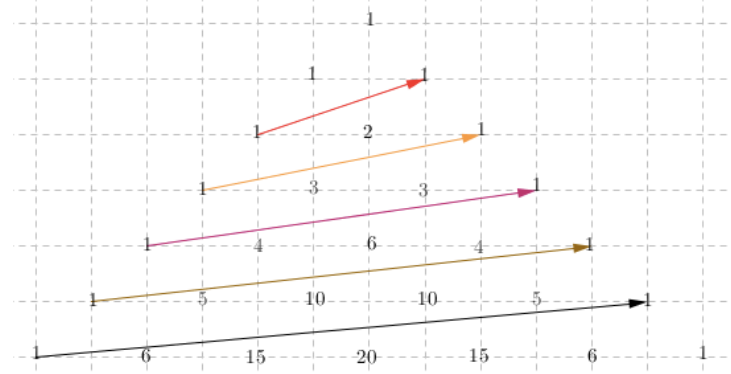

Figure 3. In this Figure different kinds of $k$-Fibonacci diagonals are represented: in red it is drawn a classical Fibonacci diagonal; in orange we find a Padovan's diagonal. Note that when $k$ increases, then all the corresponding $k$-Fibonacci diagonals reduce more and more their inclination.

Now we can give a formal description of the elements lying on each ' $k$-Fibonacci diagonal of a Pascal's triangle $\mathcal{T}$ '. To this aim recall that if $a$ is a positive real number, then by the symbol $\lfloor a\rfloor$ we will denote the integer part of a non-negative real number $a$.

Definition 2.1. Let $k \geq 0$ be an integer. For every positive integer $m$ written in the form $m=(k+1) n+j$ ( $n$ and $j$ non negative integers such that $0 \leq j \leq k)$, we will define the $m$-th $k$-Fibonacci diagonal of a Pascal's triangle $\mathcal{T}, \Delta_{k, m}$, as follows: 
If $j=0$, then we put $\Delta_{k, m}:=\{Q(n+(k-1),(k+1) \cdot 1-1) ; Q(n-1+(k-1),(k+1)$. $2-1) ; \ldots ; Q(n-i+(k-1),(k+1) \cdot(i+1)-1), \forall i$ such that $n-i+(k-1) \geq$ $(k+1)(i+1)-1\}$.

Note that in this case for describing $\Delta_{k, m}$, we must consider $i=0$, and all the positive indices $i$ such that $i \leq \frac{n-1}{k+2}$. Thus in this case the number of the elements which lie on $\Delta_{k, m}$ is $1+\left\lfloor\frac{n-1}{k+2}\right\rfloor$.

If $j \neq 0$, then we put $\Delta_{k, m}:=\{Q(n+k,(k+1) \cdot 0+(j-1)) ; Q(n-1+k,(k+1) \cdot 1+(j-$ $1)) ; \ldots ; Q(n-i+k,(k+1) i+(j-1)), \forall i$ such that $n-i+k \geq(k+1) i+(j-1)\}$.

Note that in this case for describing $\Delta_{k, m}$, we must consider 0 , and all the positive indices $i$ such that $i \leq \frac{n+1+(k-j)}{(k+2)}$. It follows in this case that the number of the elements which lie on $\Delta_{k, m}$ is: $1+\left\lfloor\frac{n+1+(k-j)}{(k+2)}\right\rfloor$. In particular if $1 \leq m<k$, then $\Delta_{k, m}=\{Q(k,(m-1))\}$.

It turns out that if we consider $k$ and $m$ as above, then the sum $S_{k, m}$ of the elements lying on the $m$-th $k$-Fibonacci diagonal $\Delta_{k, m}$ is described by the following relations:

$$
S_{k, m}= \begin{cases}\sum_{i=0}^{1+\left\lfloor\frac{n-1}{k+2}\right\rfloor}\left(\begin{array}{c}
n-i+(k-1) \\
(k+1)(i+1)-1
\end{array}\right)=\sum_{i=0}^{\left\lfloor\frac{n+k+1}{k+2}\right\rfloor}\left(\begin{array}{c}
n-i+(k-1) \\
(k+1)(i+1)-1
\end{array}\right), \text { if } j=0 ; \\
1+\left\lfloor\frac{n+k+1-j}{(k+2)}\right\rfloor\left(\begin{array}{c}
n-i+k \\
(k+1) i+(j-1)
\end{array}\right), & \text { if } j \neq 0\end{cases}
$$

The above equation (2.2) can be rewritten as follows:

$$
S_{k, m}=\sum_{i=0}^{1-\delta_{j}^{0}+\left\lfloor\frac{n+k-j+1}{k+2}\right\rfloor}\left(\begin{array}{c}
n-i+\left(k-\delta_{j}^{0}\right) \\
(k+1)\left(i+\delta_{j}^{0}\right)+j-1
\end{array}\right) \text {, where }\left\{\begin{array}{l}
m=(k+1) n+j, \\
\text { and } 0 \leq j \leq k
\end{array}\right.
$$

where for every non negative integers $a$ and $b, \delta_{a}^{b}$ denotes the Kronecker symbol:

$$
\delta_{a}^{b}=\left\{\begin{array}{ll}
1 & \text { if } a=b \\
0 & \text { if } a \neq b
\end{array} .\right.
$$

In the next section, using the equation (2.3) we will see that each $\left(S_{k, m}\right)$ is a recursive sequence (see Theorem 3.4). This gives a positive answer to the Question 1.

\section{The $k$-Padovan sequences and the generalized Lucas identity}

For every $k \geq 0$, the $k$-Padovan sequence is defined as a linear homogeneous recurrence with constant coefficients defined by the positions:

$$
\left\{\begin{array}{l}
P_{k, 1}=\left(\begin{array}{l}
k \\
0
\end{array}\right), P_{k, 2}=\left(\begin{array}{c}
k \\
1
\end{array}\right), \ldots, P_{k, k+1}=\left(\begin{array}{c}
k \\
k
\end{array}\right), \text { and } P_{k, k+2}=1 . \\
P_{k, m}=P_{k, m-(k+1)}+P_{k, m-(k+2)}, \quad m>k+2 .
\end{array}\right.
$$

Clearly, the Fibonacci sequence can be seen as the '0-Padovan sequence' as well as the Padovan sequence can be seen as the '1-Padovan sequence'.

Our main goal is to find a generalized Lucas identity, for $k$-Padovan sequences which extends both the Lucas identity (1.1) and the Padovan identity (1.3).

Before proving our main theorem we will need some technical lemmas.

Recall that, given a non negative integer $a$ and an integer $b$, the binomial coefficient $a$ choose $b$ is defined as follows (see [11, Section 11.1]). 


$$
\left\{\begin{array}{l}
\left(\begin{array}{l}
a \\
b
\end{array}\right)=\frac{a !}{(a-b) ! b !} \quad \text { if } \quad b \leq a \\
\left(\begin{array}{l}
a \\
b
\end{array}\right)=0 \text { if } \quad \text { it is either } b<0 \text { or } b>a
\end{array} .\right.
$$

In our proofs we will often use the so-called Pascal's rule (also known as Stiefel's identity):

$$
\left(\begin{array}{l}
a \\
b
\end{array}\right)+\left(\begin{array}{c}
a \\
b+1
\end{array}\right)=\left(\begin{array}{l}
a+1 \\
b+1
\end{array}\right), \quad \forall a \in \mathbb{N}_{0} \quad \text { and } b \in \mathbb{Z} .
$$

The following lemma shows that certain binomial coefficients that we are going to use (see Theorem 3.4) can be considered.

Lemma 3.1. Let $j, k, n$ be integers such that $0 \leq j \leq k, n \geq 0$ and $j+n>0$. Then, for every $i \leq 1-\delta_{j}^{0}+\left\lfloor\frac{n+k-j+1}{k+2}\right\rfloor$ the number $n-i+\left(k-\delta_{j}^{0}\right)$ is positive, and the binomial coefficient $\left(\begin{array}{c}n-i+\left(k-\delta_{j}^{0}\right) \\ (k+1)\left(i+\delta_{j}^{0}\right)+j-1\end{array}\right)$ can be considered in particular.

Proof. Assume that $k=0$, then by hypothesis $j$ is likewise 0 and for every $i$ such that $i \leq 1-\delta_{j}^{0}+\left\lfloor\frac{n+k-j+1}{k+2}\right\rfloor=\left\lfloor\frac{n+1}{2}\right\rfloor$, we have $n-i+\left(k-\delta_{j}^{0}\right)=n-i+1 \geq 0$.

Suppose now that $k>0$. By hypothesis $i \leq 1-\delta_{j}^{0}+\frac{n+k-j+1}{k+2}$, then $n-i+k-\delta_{j}^{0} \geq$ $n-1-\frac{n+k-j+1}{k+2}+k=n-2-\frac{n-j-1}{k+2}+k>n-2-\frac{n-j-1}{2}+k=(n-3+j+2 k) / 2$. This last term is positive because of $k \neq 0$ and $n+j>0$. On the other hand, the integer $(k+1)\left(i+\delta_{j}^{0}\right)+j-1$ is non-negative, so that each binomial coefficient of the statement can be considered.

Remark 3.2. Let $j, k, n$ be non negative integers such that $0 \leq j \leq k$. If $n(k+1)+j>$ $k+2$, then $n \geq 1+\delta_{j}^{0}$, so that $n-1 \geq 0$ and $j+n-1>0$. An application of Lemma 3.1 to $j, k$ and $n-1$ shows that $n-1-i+k-\delta_{j}^{0}$ is a non negative integer, for every $i \leq 1-\delta_{j}^{0}+\left\lfloor\frac{n+k-j+1}{k+2}\right\rfloor$. In particular we may consider all binomials that will appear in the statement of the following lemma.

Lemma 3.3. Let $j, k, n$ be integers such that $0 \leq j \leq k$, and $n(k+1)+j>k+2$, then the following binomial relation holds:

$$
\sum_{i=\delta_{j}^{0}\left(1-\delta_{j}^{0}\right)+\delta_{j}^{1}}^{1-\delta_{j}^{0}+\left\lfloor\frac{n+k-j}{k+2}\right\rfloor}\left(\begin{array}{c}
n-1-i+k-\delta_{j}^{0} \\
(k+1)\left(i+\delta_{j}^{0}\right)+j-1
\end{array}\right)=\sum_{i=\delta_{j}^{0}\left(1-\delta_{j}^{0}\right)+\delta_{j}^{1}}^{1-\delta_{j}^{0}+\left\lfloor\frac{n+k-j+1}{k+2}\right\rfloor}\left(\begin{array}{c}
n-1-i+k-\delta_{j}^{0} \\
(k+1)\left(i+\delta_{j}^{0}\right)+j-1
\end{array}\right) .
$$

Proof. First, we note that

$$
\frac{n+k-j}{k+2}=\left\lfloor\frac{n+k-j}{k+2}\right\rfloor+\frac{r}{k+2}, \text { where } 0 \leq r<k+2,
$$

so that $\frac{n+k-j+1}{k+2}=\left\lfloor\frac{n+k-j}{k+2}\right\rfloor+\frac{r}{k+2}+\frac{1}{k+2}$.

If $0 \leq r<k+1$ the upper indices of the sum in (3.2) coincide. In fact,

$\left\lfloor\frac{n+k-j+1}{k+2}\right\rfloor=\left\lfloor\frac{n+k-j}{k+2}\right\rfloor$. Therefore the relation (3.2) is trivially satisfied.

Assume then $r=k+1$. In this case

$$
\frac{n+k-j+1}{k+2}=\left\lfloor\frac{n+k-j}{k+2}\right\rfloor+1=\left\lfloor\frac{n+k-j+1}{k+2}\right\rfloor .
$$


It follows that in the second sum of the equation (3.2) more terms (with respect to the first sum) appear, precisely the addend related to the index

$$
\bar{i}=\frac{n+k-j+1}{k+2}+1-\delta_{j}^{0} .
$$

Note that $n+k=(k+2)\left(\bar{i}-1+\delta_{j}^{0}\right)+j-1$, and hence

$n-1-\bar{i}-\delta_{j}^{0}+k=(k+1)\left(\bar{i}+\delta_{j}^{0}\right)-1-(k+2)+j-1=(k+1)\left(\bar{i}+\delta_{j}^{0}\right)-1+j-k-3$.

It follows that the addend $\left(\begin{array}{c}n-1-\bar{i}+k-\delta_{j}^{0} \\ (k+1)\left(\bar{i}+\delta_{j}^{0}\right)+j-1\end{array}\right)$ is zero.

We are now in a position to prove our main theorem. We highlight that the statement, has been suggested by equation (2.3), which we have been derived from Pascal's triangle. In this way we will obtain a Generalized Lucas identity "via" Pascal's triangle.

Theorem 3.4 (Generalized Lucas identity). Let $k \geq 0$ be an integer, and let $\left(P_{k, m}\right)_{m \geq 1}$ be the $k$-Padovan sequence. Then $P_{k, m}=S_{k, m}$ and the following generalized Lucas identity holds:

$$
P_{k, m}=\sum_{i=0}^{1-\delta_{j}^{0}+\left\lfloor\frac{n+k-j+1}{k+2}\right\rfloor}\left(\begin{array}{c}
n-i+\left(k-\delta_{j}^{0}\right) \\
(k+1)\left(i+\delta_{j}^{0}\right)+j-1
\end{array}\right) \text {, where }\left\{\begin{array}{l}
m=(k+1) n+j, \\
\text { and } 0 \leq j \leq k
\end{array} .\right.
$$

Proof. By equation (2.3) it is sufficient to show the above identity (3.3).

Let $k \in \mathbb{N}_{0}$ and proceed by induction on $m=n(k+1)+j$.

If $m=1, \ldots, k, k+1, k+2$, then one of the following cases holds:

1) $0<j \leq k$ and $n=0$, 2) $n=1$ and $j=0$, 3) $j=1$ and $n=1$.

Note that in each of the above cases we have $1-\delta_{j}^{0}+\left\lfloor\frac{n+k-j+1}{k+2}\right\rfloor=1$.

Now, in each of these three cases we can compute the sum that appears in identity (3.3), and taking into account the definition of $P_{k, m}$, for $m=1, \ldots, k, k+1, k+2$ given by position (3.1), we have:
1) $\sum_{i=0}^{1}\left(\begin{array}{c}-i+k \\ (k+1) i+j-1\end{array}\right)=\left(\begin{array}{c}k \\ j-1\end{array}\right)+\left(\begin{array}{c}k-1 \\ k+j\end{array}\right)=\left(\begin{array}{c}k \\ j-1\end{array}\right)+0=P_{k, j}$;
2) $\sum_{i=0}^{1}\left(\begin{array}{c}1-i+(k-1) \\ (k+1) i+k\end{array}\right)=\left(\begin{array}{l}k \\ k\end{array}\right)+\left(\begin{array}{c}k-1 \\ k+1+k\end{array}\right)=1+0=P_{k, k+1}$;
3) $\sum_{i=0}^{1}\left(\begin{array}{c}1-i+k \\ (k+1) i\end{array}\right)=\left(\begin{array}{c}1+k \\ 0\end{array}\right)+\left(\begin{array}{c}k \\ k+1\end{array}\right)=1+0=P_{k, k+2}$.

Therefore we may suppose that $m>k+2$, and assume that the statement is true for $P_{k, m-(k+1)}$ and $P_{k, m-(k+2)}$.

Note that, as $m=n(k+1)+j$, where $0 \leq j \leq k$ and $m>k+2$, then $j, k, n$, satisfy all the conditions of Lemma 3.3. We will split our analysis into three cases:

a) $j=0, \quad$ b) $j=1, \quad$ c) $1<j \leq k$.

a) In this case we have $m=n(k+1)$. By definition (see (3.1))

$$
\begin{aligned}
P_{k, n(k+1)} & =P_{k, n(k+1)-(k+1)}+P_{k, n(k+1)-(k+2)} \\
& =P_{k,(n-1)(k+1)}+P_{k,(n-2)(k+1)+k},
\end{aligned}
$$

and by hypothesis of induction 


$$
\begin{aligned}
P_{k, n(k+1)} & =\sum_{i=0}^{\left\lfloor\frac{n-1+k+1}{k+2}\right\rfloor}\left(\begin{array}{c}
n-1-i+k-1 \\
(k+1)(i+1)-1
\end{array}\right)+\sum_{i=0}^{1+\left\lfloor\frac{n-2+1}{k+2}\right\rfloor}\left(\begin{array}{c}
n-2-i+k \\
(k+1) i+k-1
\end{array}\right) \\
& =\sum_{i=0}^{\left\lfloor\frac{n+k}{k+2}\right\rfloor}\left(\begin{array}{c}
n-2-i+k \\
(k+1)(i+1)-1
\end{array}\right)+\sum_{i=0}^{\left\lfloor\frac{n+k+1}{k+2}\right\rfloor}\left(\begin{array}{c}
n-2-i+k \\
(k+1)(i+1)-2
\end{array}\right) .
\end{aligned}
$$

It follows by Lemma 3.3 and Pascal's rule that

$$
P_{k, n(k+1)}=\sum_{i=0}^{\left\lfloor\frac{n+k+1}{k+2}\right\rfloor}\left(\begin{array}{c}
n-1-i+k \\
(k+1)(i+1)-1
\end{array}\right),
$$

that is what we want to prove.

b) In this case we have $m=n(k+1)+1$. By Definition 3.1 we have

$$
\begin{aligned}
P_{k, n(k+1)+1} & =P_{k, n(k+1)+1-(k+1)}+P_{k, n(k+1)+1-(k+2)} \\
& =P_{k,(n-1)(k+1)+1}+P_{k,(n-1)(k+1)},
\end{aligned}
$$

and by hypothesis of induction

$$
\begin{aligned}
P_{k, n(k+1)+1} & =\sum_{i=0}^{1+\left\lfloor\frac{n-1+k}{k+2}\right\rfloor}\left(\begin{array}{c}
n-1-i+k \\
(k+1) i
\end{array}\right)+\sum_{i=0}^{\left\lfloor\frac{n+k}{k+2}\right\rfloor}\left(\begin{array}{c}
n-1-i+k-1 \\
(k+1)(i+1)-1
\end{array}\right) \\
& =\sum_{i=0}^{1+\left\lfloor\frac{n-1+k}{k+2}\right\rfloor}\left(\begin{array}{c}
n-1-i+k \\
(k+1) i
\end{array}\right)+\sum_{i=0}^{\left\lfloor\frac{n+k}{k+2}\right\rfloor}\left(\begin{array}{c}
n-(1+i)+k-1 \\
(k+1)(i+1)-1
\end{array}\right) \\
& =\sum_{i=0}^{1+\left\lfloor\frac{n-1+k}{k+2}\right\rfloor}\left(\begin{array}{c}
n-1-i+k \\
(k+1) i
\end{array}\right)+\sum_{i=1}^{1+\left\lfloor\frac{n+k}{k+2}\right\rfloor}\left(\begin{array}{c}
n-i+k-1 \\
(k+1) i-1
\end{array}\right) \\
& =1+\sum_{i=1}^{1+\left\lfloor\frac{n-1+k}{k+2}\right\rfloor}\left(\begin{array}{c}
n-1-i+k \\
(k+1) i
\end{array}\right)+\sum_{i=1}^{1+\left\lfloor\frac{n+k}{k+2}\right\rfloor}\left(\begin{array}{c}
n-i+k-1 \\
(k+1) i-1
\end{array}\right) .
\end{aligned}
$$

It follows by Lemma 3.3 and Pascal's rule that

$P_{k, n(k+1)+1}=1+\sum_{i=1}^{1+\left\lfloor\frac{n+k}{k+2}\right\rfloor}\left(\begin{array}{c}n-i+k \\ (k+1) i\end{array}\right)=\sum_{i=0}^{1+\left\lfloor\frac{n+k}{k+2}\right\rfloor}\left(\begin{array}{c}n-i+k \\ (k+1) i\end{array}\right)$,

that is what we wanted to prove.

c) In this case we have $m=n(k+1)+1$ where $1<j<k+1$. By Definition 3.1 we have

$$
\begin{aligned}
P_{k, n(k+1)+j} & =P_{k, n(k+1)+j-(k+1)}+P_{k, n(k+1)+j-(k+2)} \\
& =P_{k,(n-1)(k+1)+j}+P_{k,(n-1)(k+1)+j-1},
\end{aligned}
$$

and by hypothesis of induction

$$
P_{k, n(k+1)+j}=\sum_{i=0}^{1+\left\lfloor\frac{n+k-j}{k+2}\right\rfloor}\left(\begin{array}{c}
n-1-i+k \\
(k+1) i+j-1
\end{array}\right)+\sum_{i=0}^{1+\left\lfloor\frac{n+k-j+1}{k+2}\right\rfloor}\left(\begin{array}{c}
n-1-i+k \\
(k+1) i+j-2
\end{array}\right) .
$$


It follows by Lemma 3.3 and Pascal's rule that

$$
P_{k, n(k+1)+j}=\sum_{i=0}^{1+\left\lfloor\frac{n+k-j+1}{k+2}\right\rfloor}\left(\begin{array}{c}
n-i+k \\
(k+1) i+j-1
\end{array}\right)
$$

The proof is completed.

Remark 3.5. Lucas identity (see (1.1)) can be obtained as particular case of Theorem 3.4 .

In fact, when $k=0$, for every positive integer $m$ we have $m=(0+1) n+0$, so that $m=n$ and $j=0$. It follows that $\delta_{j}^{0}=1$ and the equation (3.3) becames:

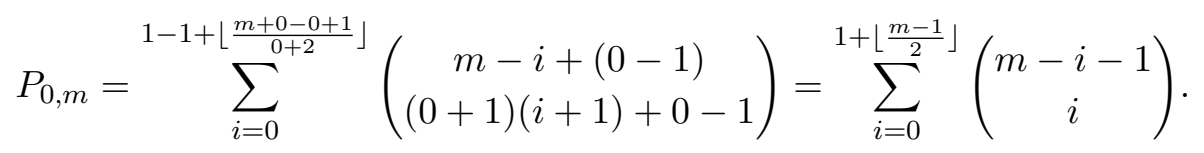

On the other hand $P_{0, m}$ is the Fibonacci sequence; therefore when $k=0$ equation (3.3) gives Lucas identity (1.1).

Remark 3.6. Padovan identity (see (1.3)) can be obtained as particular case of Theorem 3.4 .

In fact, when $k=1$, for every positive integer $m$ two cases may appear:

1) If $m$ is odd, then $m=(1+1) n+1$, and in equation (3.3) we may put $j=1$, and $\delta_{j}^{0}=0$ :

$$
\begin{aligned}
P_{1, m} & =\sum_{i=0}^{1-0+\left\lfloor\frac{n+1-1+1}{3}\right\rfloor}\left(\begin{array}{c}
n-i+(1-0) \\
(1+1)(i+0)+1-1
\end{array}\right)=\sum_{i=0}^{1+\left\lfloor\frac{n+1}{3}\right\rfloor}\left(\begin{array}{c}
n-i+1 \\
2 i
\end{array}\right) \\
& =\sum_{i=0}^{1+\left\lfloor\frac{n+1}{3}\right\rfloor}\left(\begin{array}{c}
n-i+1 \\
2 i
\end{array}\right)=\sum_{i=0}^{\left\lfloor\frac{n+1}{3}\right\rfloor}\left(\begin{array}{c}
n-i+1 \\
2 i
\end{array}\right)+0 .
\end{aligned}
$$

2) If $m$ is even, then $m=(1+1) n+0$, and in equation (3.3) we may put $j=0$, and $\delta_{j}^{0}=1$. Applying Lemma 3.3, equation (3.3) becames:

$$
\begin{aligned}
P_{1, m} & =\sum_{i=0}^{\left\lfloor\frac{n+2}{3}\right\rfloor}\left(\begin{array}{c}
n-i \\
2(i+1)-1
\end{array}\right) \\
& =\sum_{i=0}^{\left\lfloor\frac{n+3}{3}\right\rfloor}\left(\begin{array}{c}
n-i \\
2(i+1)-1
\end{array}\right)=\sum_{i=0}^{1+\left\lfloor\frac{n}{3}\right\rfloor}\left(\begin{array}{c}
n-i \\
2 i+1
\end{array}\right)=\sum_{i=0}^{\left\lfloor\frac{n}{3}\right\rfloor}\left(\begin{array}{c}
n-i \\
2 i+1
\end{array}\right)+0 .
\end{aligned}
$$

Summarizing equations (3.5) and (3.7), we have:

$$
P_{1, m}=\sum_{i=0}^{\left\lfloor\frac{n+1}{3}\right\rfloor}\left(\begin{array}{c}
n+1-i \\
2 i
\end{array}\right) \text { if } m=2 n+1 \text { and } P_{1, m}=\sum_{i=0}^{\lfloor n / 3\rfloor}\left(\begin{array}{c}
n-i \\
2 i+1
\end{array}\right) \text { if } m=2 n,
$$

that is equivalent to Padovan identity (see equation (1.3)). 
Remark 3.7. It should be noted that sequences $\left(S_{k, m}\right)$ appear to be related to the family $\left(T_{n}\right)$ of all sequences which satisfy the following equation (see equation (7) p.291, [4, Theorem 1]):

$$
T_{n}-x\left(\begin{array}{l}
r \\
1
\end{array}\right) T_{n-1}+x^{2}\left(\begin{array}{l}
r \\
2
\end{array}\right) T_{n-2}+\cdots+(-1)^{r} x^{r}\left(\begin{array}{l}
r \\
r
\end{array}\right) T_{n-r}=y^{r} T_{n-r-q} .
$$

For example, when $k=1$, then $\left(S_{1, m}\right)$ is the Padovan sequence, and by Theorem 3.4, and by equation (3.1) we have

$$
S_{1, m}=S_{1, m-2}+S_{1, m-3} .
$$

The behavior of $\left(S_{1, m}\right)$ is described by Remark 3.6, in particular we may consider the even and odd cases, which correspond to the indices of the type $m=2 n+j$ with $0 \leq j \leq 1$.

We will see that both the subsequences $A_{n}=S_{1,2 n}$ and $B_{n}=S_{1,2 n+1}$, satisfy the equation (3.9), by choosing $r=2$ and $q=x=y=1$. This is equivalent to say that for both the cases $j=0$ and $j=1$ the following relation holds:

$$
S_{1,2 n+j}=2 S_{1,2(n-1)+j}-S_{1,2(n-2)+j}+S_{1,2(n-3)+j} .
$$

Starting from the second part of the above equation, we have:

$$
\begin{aligned}
2 S_{1,2(n-1)+j}-S_{1,2(n-2)+j}+S_{1,2(n-3)+j} & \\
= & 2 S_{1, m-2}-S_{1, m-4}+S_{1, m-6} \\
= & 2 S_{1, m-2} \underbrace{-S_{1, m-4}-S_{1, m-5}}_{b y(3.10)}+\underbrace{S_{1, m-5}+S_{1, m-6}}_{b y(3.10)} \\
= & 2 S_{1, m-2} \quad-S_{1, m-2} \quad \begin{array}{c}
+S_{1, m-3} \\
=
\end{array} S_{1, m-2}+S_{1, m-3}=S_{1, m}=S_{1,2 n+j} .
\end{aligned}
$$

If $k=2$, then by Theorem 3.4, and by equation (3.1) we have

$$
S_{2, m}=S_{2, m-3}+S_{2, m-4} .
$$

Now we put $m=3 n+j$ with $0 \leq j \leq 2$, and will show that for every $j=0,1,2$, each subsequence $\left(S_{2,3 n+j}\right)_{n}$ satisfies the equation (3.9), by choosing $r=3$ and $q=x=y=1$. This is equivalent to prove that for every $j=0,1,2$ the following relation holds:

$$
S_{2,3 n+j}=3 S_{2,3(n-1)+j}-3 S_{2,3(n-2)+j}+S_{2,3(n-3)+j}+S_{2,3(n-4)+j} .
$$

Starting from the second part of the above equation, we have:

$$
\begin{aligned}
3 S_{2,3(n-1)+j} & -3 S_{2,3(n-2)+j}+S_{2,3(n-3)+j}+S_{2,3(n-4)+j} \\
& =3 S_{2,3 n+j-3}-3 S_{2,3 n+j-6}+S_{2,3 n+j-9}+S_{2,3 n+j-12} \\
& =3 S_{2, m-3}-3 S_{2, m-6}+S_{2, m-9}+S_{2, m-12} \\
& =3 S_{2, m-m}+\underbrace{+S_{2, m-9}+S_{2, m-10}}_{b y(3.11)} \underbrace{-S_{2, m-10}-S_{2, m-11}}_{b y(3.11)} \underbrace{+S_{2, m-11}+S_{2, m-12}}_{b y(3.11)} \\
& =3 S_{2, m-7} \quad \begin{array}{c}
+S_{2, m-7} \\
+S_{2, m-m}
\end{array} \\
& =2 S_{2, m-7}+S_{2, m-6}+S_{2, m-8} \\
& =S_{2, m-3}+S_{2, m-4} \\
& =S_{2, m}=S_{2,3 n+j} .
\end{aligned}
$$

Similarly, in the general case $(k>1)$, if we put $m=(k+1) n+j$, where $0 \leq j \leq k$, we may conjecture that each subsequence $\left(S_{2,(k+1) n+j}\right)_{n}$ satisfy the equation (3.9), by choosing $r=k+1$ and $q=x=y=1$. 


\section{Conclusions}

The idea to discover numerical harmony inside geometric configurations is one of the main goals for mathematics and, as a general problem, one could ask which recursive sequences can be described through geometrical objects.

It is known that recursive sequences may have applications in counting problems referred to graphs, and perhaps also it should be mentioned that recursive sequences are useful in GPS systems (for localization). Here we may expect that other kind of Padovan-like sequences may be useful both in architecture and design especially in digital process and design [18], as well as in a large spectrum of problems in mathematics (see [21]).

Acknowledgment. We wish to thank the anonymous referees for their suggestions and comments. This work was supported by the National Group for Algebraic and Geometric Structures, and their Applications (GNSAGA -INdAM), Ministry of Italian Research.

\section{References}

[1] Z. Akyuz and S. Halici, On some combinatorial identities involving the terms of generalized Fibonacci and Lucas sequences, Hacet. J. Math. Stat. 42 (4), 431-435, 2013.

[2] G. Anatriello and G. Vincenzi, Tribonacci-like sequences and generalized Pascal's triangles, Internat. J. Math. Ed. Sci. Tech. 45 (8), 1220-1232, 2014.

[3] G. Anatriello and G. Vincenzi, Padovan-like sequences and generalized Pascal's Triangles, An. Ştiinţ. Univ. Al. I. Cuza Iaşi, LXVI (f1), 25-35, 2020.

[4] H. Belbachir, T. Komatsu and L. Szalay, Linear recurrences associated to rays in Pascal's triangle and combinatorial identities, Math. Slovaca 64, 287-300, 2014.

[5] J.H. Conway and R.K. Guy, The Book of Numbers, World Scientific, Singapore, 2008.

[6] A. Fiorenza and G. Vincenzi, Limit of ratio of consecutive terms for general order- $k$ linear homogeneous recurrences with constant coefficients, Chaos Solitons Fractals 44, 147-152, 2011.

[7] N. Gogin and A. Mylläri, Padovan-like sequences and Bell polynomials, Math. Comput. Simulation 125, 168-177, 2016.

[8] S. Halici and A. Karataş, On a generalization for Fibonacci quaternions, Chaos Solitons Fractals 98, 178-182, 2017.

[9] A. Ipek, On $(p, q)$-Fibonacci quaternions and their Binet's Formulas, generating functions and certain binomial sums, Adv. Appl. Clifford Algebras 27 (2), 1343-1351, 2017.

[10] G. Kallós, A generalization of Pascal's triangle using powers of base numbers, Ann. Math. Blaise Pascal 13 (1), 1-15, 2006.

[11] T. Koshy, Fibonacci and Lucas Numbers with Applications, Wiley-Interscience, New York, 2001.

[12] L. Maronhič, Plastic Number: Construction and Applications, 5th Virtual International Conference on Advanced Research in Scientific Areas (ARSA-2016) Slovakia, November 7-11, 2016.

[13] L. Németh, The trinomial transform triangle, J. Integer Seq. 21 (7), Art. 18.7.3, pp18, 2018.

[14] L. Németh and L. Szalay, Recurrence sequences in the hyperbolic Pascal triangle corresponding to the regular mosaic 4, 5, Ann. Math. Inform. 46, 165-173, 2016.

[15] M.J. Ostwald, Under siege: the golden mean in architecture, Nexus Netw. J. 2, 75-81, 2000.

[16] R. Padovan, Proportion: Science, Philosophy, Architecture,1-st Edition, Taylor and Francis, London and New York, 1999. 
[17] R. Padovan, Dom Hans van der Laan and the Plastic Number, in:Williams K., Ostwald M. (eds) Architecture and Mathematics from Antiquity to the Future, Birkhäuser, 407-419, 2015.

[18] P. Szalapaj, Contemporary Architecture and the Digital Design Process, RoutledgeArchitectural press, New York, 2005.

[19] A. Szynal-Liana and I. Wloch, The Fibonacci hybrid numbers, Util. Math. 110, 3-10, 2019.

[20] A.G. Shannon, Tribonacci numbers and Pascal's Pyramid, Fibonacci Quart. 15, 268$275,1977$.

[21] A.G. Shannon, P.G. Anderson and A.F. Horadam, Properties of Cordonnier, Perrin and van der Laan numbers, Int. J. Math. Educ. Sci. Technol. 37 (7), 825-831, 2006.

[22] M.Z. Spivey, The Art of Proving Binomial Identities, CRC Press (Taylor and Francis Group), A. Chapman and Hall Book. Boca Raton (FL), 2019.

[23] G. Vincenzi and S. Siani, Fibonacci-like sequences and generalized Pascal's triangles. Int. J. Math. Educ. Sci. Technol. 45 (4), 609-614, 2014.

[24] S.-L. Yang, Some identities involving the binomial sequences, Discrete Math. 308 (1), 51-58, 2008. 